\title{
Abelianization of QCD in the Maximally Abelian Gauge and the Nambu-'t Hooft Picture for Color Confinement
}

\author{
H. Suganuma ${ }^{\mathrm{a}}$ and H. Ichie ${ }^{\mathrm{b}}$ \\ ${ }^{\text {a }}$ Faculty of Science, Tokyo Institute of Technology, Ohokayama 2-12-1, Meguro, Tokyo 152-8551, Japan \\ b Humboldt Univ. zu Berlin, Institut für Physik, Invalidenstrasse 110, D-10115 Berlin, Germany
}

We study the Nambu-'t Hooft picture for color confinement in terms of the abelianization of QCD and monopole condensation in the maximally abelian (MA) gauge. In the MA gauge in the Euclidean metric, the off-diagonal gluon amplitude is strongly suppressed, and then the off-diagonal gluon phase shows strong randomness, which leads to rapid reduction of the off-diagonal gluon correlation. In $\mathrm{SU}(2)$ and $\mathrm{SU}(3)$ lattice QCD in the MA gauge with the abelian Landau gauge, the Euclidean gluon propagator indicates a large effective mass of the off-diagonal gluon as $M_{\text {off }} \simeq 1 \mathrm{GeV}$ in the intermediate distance as $0.2 \mathrm{fm} \leq r \leq 0.8 \mathrm{fm}$. Due to the infrared inactiveness of offdiagonal gluons, infrared QCD is well abelianized like nonabelian Higgs theories in the MA gauge. We investigate the inter-monopole potential and the dual gluon field $B_{\mu}$ in the MA gauge, and find longitudinal magnetic screening with $m_{B} \simeq 0.5 \mathrm{GeV}$ in the infrared region, which indicates the dual Higgs mechanism by monopole condensation. We define the "gluonic Higgs scalar field" providing the MA projection, and find the correspondence between its hedgehog singularity and the monopole location in lattice QCD.

\section{Dual Superconductor Theory for Color Confinement and QCD in MA Gauge}

To understand color confinement is one of the most difficult problems remaining in particle physics [1]. As the Regge trajectories and lattice QCD indicate, quark confinement is characterized by one-dimensional squeezing of the color-electric flux with the string tension $\sigma \simeq 1 \mathrm{GeV} / \mathrm{fm}$. On the confinement mechanism, Nambu proposed the dual superconductor theory in 1974, based on the electro-magnetic duality [2]. In this theory, there occurs the one-dimensional squeezing of the color-electric flux by the dual Meissner effect due to condensation of bosonic color-magnetic monopoles. But, there are two large gaps between QCD and the dual superconductor theory.

1. The dual superconductor theory is based on the abelian gauge theory subject to the Maxwell-type equations, where electromagnetic duality is manifest, while QCD is a nonabelian gauge theory.

2. The dual superconductor theory requires color-magnetic monopole condensation as the key concept, while QCD itself does not have color-magnetic monopoles.
These gaps may be filled simultaneously by taking maximally abelian (MA) gauge fixing, which reduces QCD to an abelian gauge theory including color-magnetic monopoles [3].

In Euclidean QCD, the MA gauge is defined so as to minimize the "total amount" of the offdiagonal gluon amplitude $[4,5]$,

$$
R_{\text {off }}\left[A_{\mu}(\cdot)\right] \equiv \int d^{4} x \operatorname{tr}\left\{\left[\hat{D}_{\mu}, \vec{H}\right]\left[\hat{D}_{\mu}, \vec{H}\right]^{\dagger}\right\}
$$

by the $\mathrm{SU}\left(N_{c}\right)$ gauge transformation. Since the $\mathrm{SU}\left(N_{c}\right)$ covariant derivative $\hat{D}_{\mu} \equiv \hat{\partial}_{\mu}+i e A_{\mu}$ obeys the adjoint gauge transformation, the local form of the MA gauge condition is easily derived as $\left[\vec{H},\left[\hat{D}_{\mu},\left[\hat{D}_{\mu}, \vec{H}\right]\right]\right]=0$.

In the MA gauge, the nonabelian gauge symmetry is partially fixed as $\mathrm{SU}\left(N_{c}\right)_{\text {local }} \rightarrow \mathrm{U}(1)_{\text {local }}^{N_{c}-1} \times$ Weyl ${ }_{N_{c}}^{\text {global }}$, and QCD reduces into an abelian gauge theory, where the off-diagonal gluons behave as charged matter fields providing the colorelectric current in terms of the residual abelian gauge symmetry. In the MA gauge, according to the reduction of the nonabelian gauge manifold, color-magnetic monopoles appear as the topological defects reflecting the nontrivial homotopy group $\Pi_{2}\left(\mathrm{SU}\left(N_{c}\right) / \mathrm{U}(1)^{N_{c}-1}\right)=\Pi_{1}\left(\mathrm{U}(1)^{N_{c}-1}\right)=$ 
$\mathbf{Z}_{\infty}^{N_{c}-1}$, as 't Hooft [3] pointed out with the similar logic on the 't Hooft-Polyakov monopole.

Thus, in the MA gauge, $Q C D$ reduces into an abelian gauge theory including color-magnetic monopoles, and this situation is conjectured to provide a realistic dual-superconductor picture for the confinement mechanism based on QCD, which we call here the Nambu-'t Hooft picture.

\section{Strong Randomness of Off-diagonal Gluon Phases in the MA Gauge and Its Physical Implications}

To find out essence of the MA gauge on abelianization, we study the off-diagonal gluon field $A_{\mu}^{ \pm}(x) \equiv \frac{1}{\sqrt{2}}\left(A_{\mu}^{1} \pm i A_{\mu}^{2}\right)=e^{ \pm i \chi_{\mu}(x)}\left|A_{\mu}^{ \pm}(x)\right|$ in the MA gauge in $\mathrm{SU}(2)$ lattice QCD, and find the two remarkable features as follows $[4,5]$.

1. The off-diagonal gluon amplitude $\left|A_{\mu}^{ \pm}(x)\right|$ is strongly suppressed by $\mathrm{SU}\left(N_{c}\right)$ gauge transformation in the MA gauge.

2. The off-diagonal gluon phase $\chi_{\mu}(x)$ tends to be random, because $\chi_{\mu}(x)$ is not constrained by MA gauge fixing at all, and only the constraint from the QCD action is weak due to a small accompanying factor $\left|A_{\mu}^{ \pm}\right|$.

Owing to the strong randomness of the offdiagonal gluon phase, we expect strong cancellation of the off-diagonal gluon contribution in the MA gauge, especially for the infrared quantities.

For instance, within the random-variable approximation for the off-diagonal gluon phase $\chi_{\mu}(s)$ in the MA gauge, we analytically derive perfect abelian dominance of the string tension, $\sigma_{\mathrm{SU}(2)}=\sigma_{\mathrm{Abel}}$, via the perimeter law of the offdiagonal gluon contribution in the Wilson loop as

$$
\begin{aligned}
W_{C}^{\text {off }} & \equiv\left\langle W_{C}\left[A_{\mu}^{a}\right]\right\rangle /\left\langle W_{C}\left[A_{\mu}^{ \pm} \equiv 0, A_{\mu}^{3}\right]\right\rangle_{\mathrm{MA}} \\
& \simeq \exp \left\{-L a^{2}\left\langle\left|e A_{\mu}^{ \pm}\right|^{2}\right\rangle_{\mathrm{MA}} / 4\right\},
\end{aligned}
$$

where $\left\langle W_{C}\left[A_{\mu}^{a}\right]\right\rangle$ denotes the $\mathrm{SU}(2)$ Wilson loop, and $\left\langle W_{C}\left[A_{\mu}^{ \pm} \equiv 0, A_{\mu}^{3}\right]\right\rangle_{\mathrm{MA}}$ the abelian Wilson loop in the MA gauge. We check this relation between the macroscopic quantity $W_{C}^{\text {off }}$ and the microscopic quantity $\left\langle\left|e A_{\mu}^{ \pm}\right|^{2}\right\rangle_{\mathrm{MA}}$ in lattice QCD $[4,5]$.

As another physical implication, strong randomness of off-diagonal gluon phases in the MA
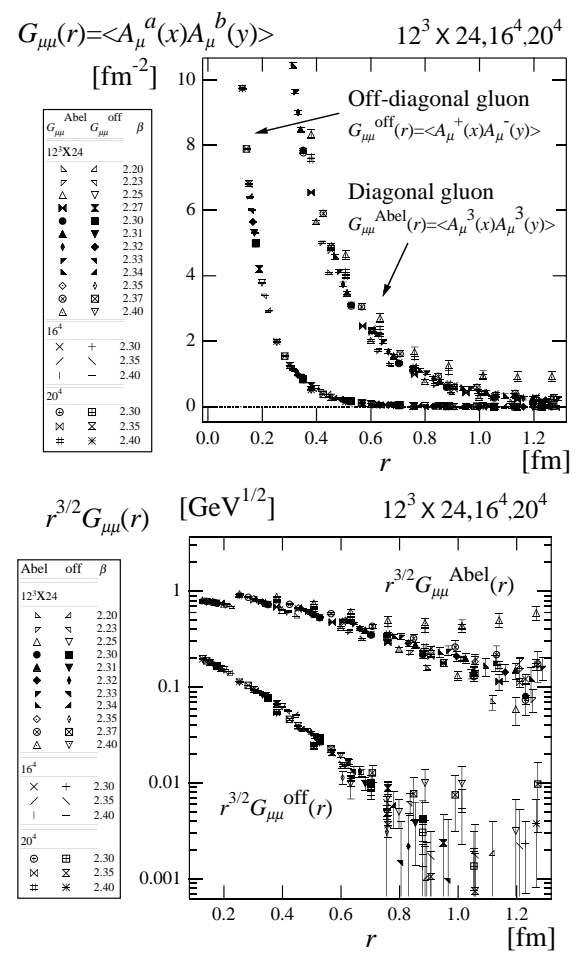

Figure 1. (a) The scalar-type gluon propagator $G_{\mu \mu}^{a}(r)$ v.s. 4-dim. distance $r$ in the MA gauge in $\mathrm{SU}(2)$ lattice QCD with $2.2 \leq \beta \leq 2.4,12^{3} \times 24$, $16^{4}, 20^{4}$. (b) The logarithmic plot of $r^{3 / 2} G_{\mu \mu}^{a}(r)$.

gauge leads to rapid reduction of off-diagonal gluon correlations [5] as

$$
\begin{aligned}
\left\langle A_{\mu}^{+}(x) A_{\nu}^{-}(y)\right\rangle_{\mathrm{MA}} & =\left\langle\left|A_{\mu}^{+}(x) A_{\nu}^{-}(y)\right| e^{i\left\{\chi_{\mu}(x)-\chi_{\nu}(y)\right\}}\right\rangle \\
& \sim\left\langle\left|A_{\mu}^{ \pm}(x)\right|^{2}\right\rangle_{\mathrm{MA}} \delta_{\mu \nu} \delta^{4}(x-y),(3)
\end{aligned}
$$

which physically indicates a large effective mass of the off-diagonal gluon in the MA gauge.

\section{Large Effective-Mass Generation of Off- diagonal Gluons in MA Gauge : Essence of Infrared Abelianization of QCD}

We study the Euclidean gluon propagator in the MA gauge $[5,6]$ using $\mathrm{SU}\left(N_{c}\right)$ lattice QCD with $N_{c}=2,3$. On the residual abelian gauge symmetry, we take the abelian Landau gauge, where the gluon configuration becomes maximally continuous under the MA gauge constraint.

We show the scalar-type gluon propagators $G_{\mu \mu}^{a}(r) \equiv\left\langle A_{\mu}^{a}(x) A_{\mu}^{a}(y)\right\rangle\left(a=1,2, . ., N_{c}^{2}-1\right)$ as 

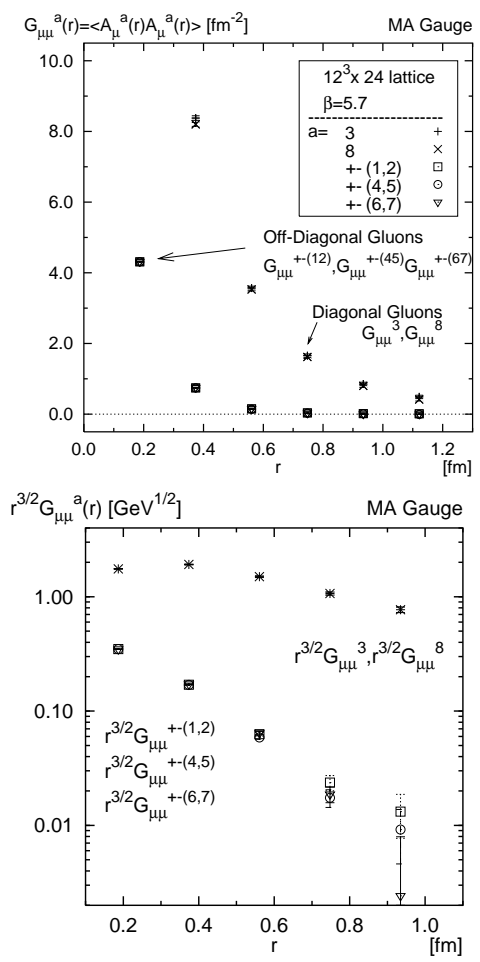

Figure 2. (a) The scalar-type gluon propagator $G_{\mu \mu}^{a}(r)$ v.s. the four-dimensional distance $r$ in the MA gauge with $\mathrm{U}(1)_{3} \times \mathrm{U}(1)_{8}$ Landau gauge fixing in $\mathrm{SU}(3)$ lattice QCD with $\beta=5.7$ and $12^{3} \times 24$. (b) The logarithmic plot of $r^{3 / 2} G_{\mu \mu}^{a}(r)$.

the function of the four-dimensional Euclidean distance $r \equiv \sqrt{\left(x_{\mu}-y_{\mu}\right)^{2}}$ in Figs.1 and 2. Both in $\mathrm{SU}(2)$ and $\mathrm{SU}(3) \mathrm{QCD}$, we find infrared abelian dominance for the gluon propagator in the $M A$ gauge: while the diagonal gluon, $A_{\mu}^{3}(x)$ or $A_{\mu}^{8}(x)$, propagates over the long distance, off-diagonal gluons propagate only within a short distance.

Next, we evaluate the effective off-diagonal gluon mass in the MA gauge from the slope analysis of the lattice QCD data of $\ln \left\{r^{3 / 2} G_{\mu \mu}(r)\right\}$, since the four-dimensional Euclidean propagator of the massive vector boson with the mass $M$ takes a Yukawa-type asymptotic form as

$G_{\mu \mu}(r) \simeq \frac{3}{4 \pi^{2}} \frac{M}{r} K_{1}(M r) \simeq \frac{3 M^{1 / 2}}{2(2 \pi)^{3 / 2}} \frac{e^{-M r}}{r^{3 / 2}}$.

As a remarkable fact, the off-diagonal gluon behaves as a massive vector field with a large constant effective mass $M_{\text {off }}$ in the intermediate dis- tance as $0.2 \mathrm{fm} \leq r \leq 0.8 \mathrm{fm}$ in the MA gauge:

1. $M_{\text {off }} \simeq 1.2 \mathrm{GeV}$ in $\mathrm{SU}(2)$ lattice $\mathrm{QCD}$ with $2.2 \leq \beta \leq 2.4$ and $12^{3} \times 24,16^{4}, 20^{4}$ (Fig. 1 ).

2. $M_{\text {off }} \simeq 1.1 \mathrm{GeV}$ in $\mathrm{SU}(3)$ lattice $\mathrm{QCD}$ with $\beta=5.7(a \simeq 0.19 \mathrm{fm})$ and $12^{3} \times 24$ (Fig.2).

Thus, both in $\mathrm{SU}(2)$ and $\mathrm{SU}(3)$ QCD, the offdiagonal gluon seems to acquire a large effective mass $M_{\mathrm{off}} \simeq 1 \mathrm{GeV}$ in the $M A$ gauge, which would be essence of infrared abelian dominance $[5,6]$.

\section{Inter Monopole Potential, Longitudinal Magnetic Screening, Infrared Monopole Condensation and Monopole Structure}

Using SU(2) lattice QCD, we study the intermonopole potential and the dual gluon propagator in the monopole part in the MA gauge, and show longitudinal magnetic screening in the infrared region, as a direct evidence of the dual Higgs mechanism by monopole condensation. The dual gluon mass is estimated as $m_{B} \simeq 0.5 \mathrm{GeV}$ [5]. Then, lattice QCD in the MA gauge exhibits infrared abelian dominance and infrared monopole condensation, which lead to the dual Ginzburg-Landau (DGL) theory [7] for infrared QCD.

Using SU(2) lattice QCD, we find the structure of the monopole in the MA gauge like the 't Hooft-Polyakov monopole: a large off-diagonal gluon amplitude around its center, as shown in Fig.3. At a large scale, off-diagonal gluons inside monopoles become invisible, and monopoles can be regarded as point-like Dirac monopoles [4,5].

Abelian magnetic monopole

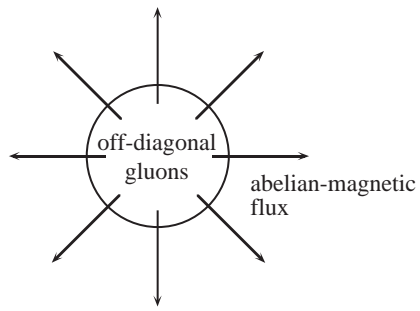

Figure 3. The schematic figure of the structure of the monopole in the MA gauge. The off-diagonal gluon amplitude is large around its center as well as the abelian magnetic field. 


\section{Monopole in MA Gauge and Hedgehog Singularity of the Gluonic Higgs Field}

To clarify the similarity between QCD in the MA gauge and the NAH theory, we introduce the "gluonic Higgs scalar field" $\vec{\phi}(x) \equiv \Omega(x) \vec{H} \Omega^{\dagger}(x)$ with $\Omega(x) \in \mathrm{SU}\left(N_{c}\right)$ so as to minimize

$R[\vec{\phi}(\cdot)] \equiv \int d^{4} x \operatorname{tr}\left\{\left[\hat{D}_{\mu}, \vec{\phi}(x)\right]\left[\hat{D}_{\mu}, \vec{\phi}(x)\right]^{\dagger}\right\}$

for arbitrary given Euclidean gluon field $\left\{A_{\mu}(x)\right\}$. The gluonic Higgs scalar $\vec{\phi}(x)$ physically corresponds to a "color-direction" of the nonabelian gauge connection $\hat{D}_{\mu}$ averaged over $\mu$ at each $x$.

Similar to $\hat{D}_{\mu}, \vec{\phi}(x)$ obeys the adjoint gauge transformation, and $\vec{\phi}(x)$ is diagonalized in the MA gauge. Therefore, $\vec{\phi}(x)$ behaves as the Higgs scalar in the NAH theory, and the hedgehog singularity of $\vec{\phi}(x)$ provides the monopole in the MA gauge. This correlation is observed in lattice QCD, when the gluon field is continuous as in the $\mathrm{SU}(2)$ Landau gauge, as shown in Fig.4 $[4,5]$.

Through the projection along $\vec{\phi}(x)$, one can extract the abelian $\mathrm{U}(1)^{N_{c}-1}$ sub-gauge-manifold close to the original $\mathrm{SU}\left(N_{c}\right)$ gauge manifold. This projection is manifestly gauge invariant and is equivalent to the ordinary MA projection. Hence, infrared relevance of the gluon mode along the color-direction $\vec{\phi}(x)$ is observed [4,5].

Acknowledgement H.S. would like to thank Prof. G. 't Hooft and Prof. Yoichiro Nambu for their valuable comments and discussions.

\section{REFERENCES}

1. For instance, Quantum Chromodynamics and Color Confinement, (World Scientific, 2001).

2. Y. Nambu, Phys. Rev. D10 (1974) 4262.

3. G. 't Hooft, Nucl. Phys. B190 (1981) 455.

4. H. Ichie, H. Suganuma, Phys.Rev.D60 (1999) 77501; Nucl.Phys.B548 (99) 365; B574 (00) 70.

5. H. Suganuma et al., Nucl. Phys. A670 (2000) 40; Prog. Theor. Phys. Suppl. 131 (1998) 559; Nucl. Phys. B (Proc. Suppl.) 106 (2002) 679.

6. K. Amemiya and H. Suganuma, Phys. Rev. D60 (1999) 114509.

7. H. Suganuma, S. Sasaki and H. Toki, Nucl. Phys. B435 (1995) 207.

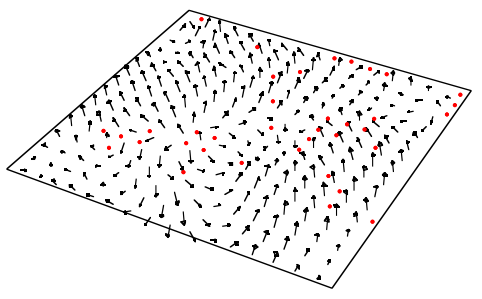

Figure 4. The gluonic Higgs scalar field $\phi(x)=$ $\phi^{a}(x) \frac{\tau^{a}}{2}$ in the $\mathrm{SU}(2)$ Landau gauge in $\mathrm{SU}(2)$ lattice QCD with $\beta=2.4$ and $16^{4}$. The arrow denotes $\left(\phi^{1}(x), \phi^{2}(x), \phi^{3}(x)\right)$. The monopoles (dots) in the MA gauge appear at the hedgehog singularities of the gluonic Higgs scalar $\phi(x)$.

\section{DISCUSSIONS}

As 't Hooft pointed out, if the dual superconductor picture is true, the off-diagonal colorcharges are to be confined, and the off-diagonal gluon correlation should be cut in the infrared limit. This tendency is observed in Figs. 1 and 2 as the rapid vanishing of off-diagonal gluon correlators in $r \geq 1 \mathrm{fm}$. This infrared screening would occur due to the off-diagonal color-charge confinement and the off-diagonal gluon pair creation.

So, the effective off-diagonal gluon mass $M_{\text {off }} \simeq$ $1 \mathrm{GeV}$ in the MA gauge is to be interpreted as the "constituent mass" in the intermediate distance, $0.2 \mathrm{fm} \leq r \leq 0.8 \mathrm{fm}$, as 't Hooft suggested.

As an interesting possibility, we conjecture the mutual relation among the localization of the offdiagonal gluon correlation, abelian monopole condensation and the off-diagonal color-charge confinement in the MA gauge as shown in Fig.5.

\section{QCD in the MA gauge}

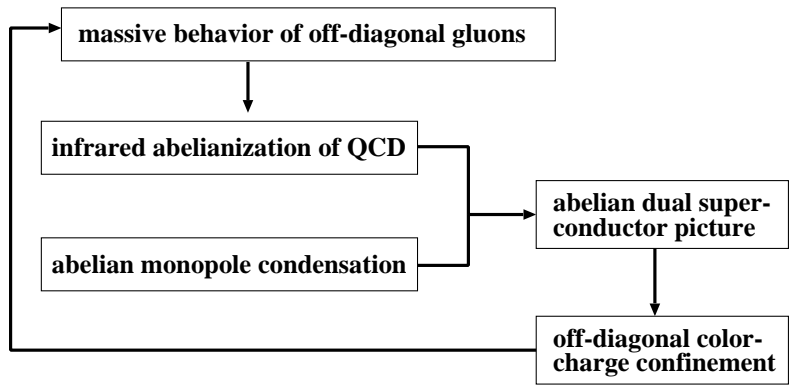

Figure 5. The conjecture on the relation between abelianization of QCD and the off-diagonal colorcharge confinement in the MA gauge. 